\title{
INDUCTION THEOREMS ON THE STABLE RATIONALITY OF THE CENTER OF THE RING OF GENERIC MATRICES
}

\author{
ESTHER BENEISH
}

\begin{abstract}
Following Procesi and Formanek, the center of the division ring of $n \times n$ generic matrices over the complex numbers $\mathbf{C}$ is stably equivalent to the fixed field under the action of $S_{n}$, of the function field of the group algebra of a $Z S_{n}$-lattice, denoted by $G_{n}$. We study the question of the stable rationality of the center $C_{n}$ over the complex numbers when $n$ is a prime, in this module theoretic setting. Let $N$ be the normalizer of an $n$-sylow subgroup of $S_{n}$. Let $M$ be a $Z S_{n}$-lattice. We show that under certain conditions on $M$, inductionrestriction from $N$ to $S_{n}$ does not affect the stable type of the corresponding field. In particular, $\mathbf{C}\left(G_{n}\right)$ and $\mathbf{C}\left(Z G_{Z_{N}} G_{n}\right)$ are stably isomorphic and the isomorphism preserves the $S_{n}$-action. We further reduce the problem to the study of the localization of $G_{n}$ at the prime $n$; all other primes behave well. We also present new simple proofs for the stable rationality of $C_{n}$ over $\mathbf{C}$, in the cases $n=5$ and $n=7$.
\end{abstract}

\section{INTRODUCTION}

The question of rationality of the center of the ring of generic matrices has been studied extensively over some decades, in particular for its connections to other problems. Geometrically, the center $C_{n}$ of $2 n \times n$ generic matrices over an algebraically closed field is the function field of an algebraic variety under a reductive group, namely $\left(M_{n} \times M_{n}\right) / \mathrm{PGL}_{n}$. The classification of quotients under connected reductive groups is one of the main open problems in invariant theory. In the Brauer group setting the stable rationality of this center implies the MerkurjevSuslin result, which says that the Brauer group of a field $k$ is generated by cyclics, provided $k$ has enough roots of unity. Le Bruyn presents an informative survey of the problem and its applications in [LL].

In 1883 Sylvester showed that for $n=2, C_{2}$ was rational over $\mathbf{C}$, the complex numbers, [S]. In 1979 and 1980 Formanek showed that $C_{n}$ was rational over $\mathbf{C}$ for the case $n=3,[\mathrm{~F} 1]$, and $n=4,[\mathrm{~F} 2]$. In 1984, Saltman proved that for all primes $n, C_{n}$ is retract rational over $\mathbf{C}$, [SD]. In 1991 Bessenrodt and Le Bruyn showed stable rationality for the cases $n=5$ and 7 [BL]. Their proof, however, requires the use of a computer. In this paper we present simple proofs for the case $n=5$ and 7, Theorems 3.3 and 3.4.

We study the problem in its module theoretic setting. If $G$ is a finite group, and $M$ is a $Z G$-lattice, then $\mathbf{C}[M]$ denotes the group algebra of the abelian group $M$, and $\mathbf{C}(M)$ its quotient field. Define the following $Z S_{n}$-lattices. $U_{n}=\sum_{i=1, \ldots, n} Z u_{i}$, where $g u_{i}=u_{g(i)}$ for all $g$ in $S_{n}$. A, usually denoted by $A_{n-1}$, is the kernel of the

Received by the editors September 22, 1996.

1991 Mathematics Subject Classification. Primary 13A50, 20C10. 
augmentation map $\varepsilon: U_{n} \rightarrow Z$. $V_{n}=\sum_{i, j, i \neq j} Z y_{i j}$ where $g y_{i j}=y_{g(i) g(j)}$ for all $g$ in $S_{n} . G_{n}$ is defined by the sequence $0 \rightarrow G_{n} \rightarrow V_{n} \rightarrow A \rightarrow 0$, where the map $V_{n} \rightarrow A$ is given by $y_{i j} \rightarrow u_{i}-u_{j}$. Procesi-Formanek show that $C_{n} \cong$ $\mathbf{C}\left(G_{n} \oplus U_{n} \oplus U_{n}\right)^{S n},\left[\mathrm{~F} 1\right.$, Theorem 3]. This implies that $C_{n}$ is stably isomorphic to $\mathbf{C}\left(G_{n}\right)^{S n}$ [L, Proposition 1.4]. Let $n$ be an odd prime. Let $H$ be an $n$-sylow subgroup of $G$, thus $H$ is cyclic of order $n$. The normalizer of $H$ in $S_{n}$, which we denote by $N$, is the semidirect product of $H$ by a cyclic group $C$ of order $n-1$.

The main result of this paper is:

Theorem 2.6. The fields $\mathbf{C}\left(G_{n}\right)$ and $\mathbf{C}\left(Z G \otimes_{Z N} G_{n}\right)$ are stably isomorphic, and the isomorphism respects the $S_{n}$-actions. The center $C_{n}$ is stably isomorphic to their fields of invariants.

This theorem is a direct consequence of Proposition 2.4, which says that under certain conditions, induction - restriction from $N$ to $G$ does not affect the stable type of the field. This is a crucial reduction step for the problem. It implies that the stable rationality of $C_{n}$ depends on the structure of $G_{n}$ as a $Z N$-lattice. Set $n=p$, and let $Z_{p}$ denote the localization of $Z$ at the prime $p$.

The second result is:

Theorem 2.7. Let $D$ and $R$ be invertible $Z N$-lattices. Suppose that $R$ is either 0 , or stably permutation and $Z_{q} C$-projective for all primes $q$ dividing $p-1$. If $\left(G_{n} \oplus R\right)_{p} \cong D_{p}$, then the fields $\mathbf{C}\left(G_{n}\right)$ and $\mathbf{C}\left(Z G \otimes_{Z N} D\right)$ are stably isomorphic as $G$-fields.

This theorem says that the stable rationality of $C_{n}$ in fact depends on the structure of $\left(G_{n}\right)_{p}$ as a $Z_{p} N$-lattice.

I would like to thank Professors John Moody, Robert Guralnick and David Saltman, for their support and advice.

1.

Let $G$ be a finite group, a $Z G$-lattice is finitely generated $Z$-free $Z G$-module. A permutation $Z G$-module is a $Z G$-lattice with a $Z$-basis permutated by $G$. An invertible $Z G$-module is a direct summand of a permutation module. A $Z G$-module $M$ is stably permutation if there exists permutation $Z G$-modules $P$ and $P^{\prime}$, such that $M \oplus P \cong P^{\prime}$.

Definition. A $Z G$-lattice $M$ is $G$-faithful if the map $G \rightarrow \operatorname{Aut}_{Z}(M)$ is injective.

\section{Notation.}

(1) For a $Z G$-lattice $M$ we denote by $M^{*}=\operatorname{Hom}_{Z}(M, Z)$, its dual.

(2) For any prime $q$ in $Z, Z_{q}$ denotes the localization of $Z$ at the ideal $(q)$, and $M_{q}=Z_{q} \otimes M$.

(3) In the remainder of this paper we will denote $S_{n}$ by $G$, and $n=p$ will be an odd prime, unless otherwise specified.

The following observations were made in [BL].

The map: $V_{n} \rightarrow A \otimes U_{n}$

$$
y_{i j} \rightarrow\left(u_{i}-u_{j}\right) \otimes u_{i}
$$

is a $Z G$-isomorphism.

Now tensoring the exact sequence

$$
0 \rightarrow A \rightarrow U_{n} \rightarrow Z \rightarrow 0
$$


by $A$, we get

$$
0 \rightarrow A \otimes A \rightarrow U_{n} \otimes A \rightarrow A \rightarrow 0
$$

The map $U_{n} \otimes A \rightarrow A$ sends $y_{i j}$ to $u_{i}-u_{j}$, so $G_{n} \cong A \otimes A$.

Notation. We let $B=A^{*} \otimes A$.

This lattice is used throughout the paper, in particular if let $Q$ be the field of rational numbers, then $Q B \cong Q G_{n}$.

The following proposition was proved by Bessenrodt and Le Bruyn, but not published. We present here a proof.

Proposition 1.1. We have the following isomorphisms of $Z G$-lattices $B \oplus U_{n} \cong$ $V_{n} \oplus Z$. Hence $B$ is stably permutation.

Proof. The sequence $0 \rightarrow A \rightarrow U_{n} \rightarrow Z \rightarrow 0$ splits when localized at all primes $q$ different from $n$, dividing the order of $G$, with splitting map given by $1 \rightarrow$ $\left(\sum_{i-1}^{n} u_{i}\right) / n$. So we have

$$
Z_{q} \oplus A_{q} \cong\left(U_{n}\right)_{q} \text { and taking duals } Z_{q} \oplus A_{q}^{*} \cong\left(U_{n}\right)_{q}
$$

Now tensoring by $A_{q}$ we get

$$
B_{q} \oplus A_{q} \cong\left(V_{n}\right)_{q}
$$

So $B_{q}$ is $Z_{q} G$-invertible. In particular $B_{q}$ is $Z_{q} P$-invertible for all $q$-sylow subgroups $P$ of $G$. To show that $B$ is invertible it suffices by [CT, Lemma 9 , section 1] to show that $B_{p}$ is $Z_{p} H$-invertible for a $p=n$-sylow subgroup of $G$. Now consider the diagram

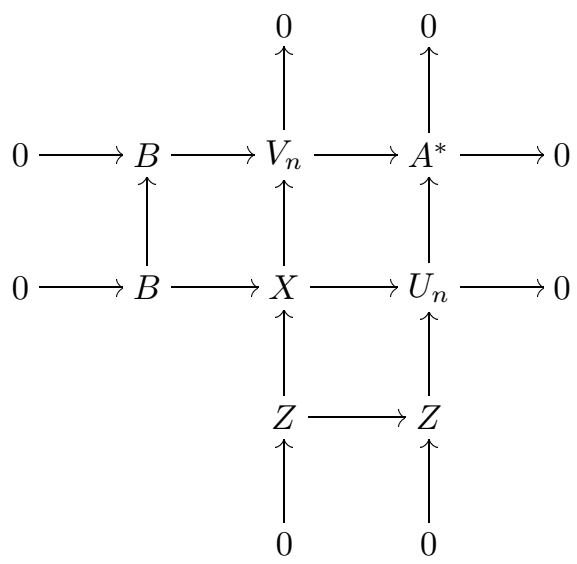

$\left(U_{n}\right)_{p} \cong Z_{p} S_{n} / S_{n-1}$ and $\left(V_{n}\right)_{p} \cong Z_{p} S_{n} / S_{n-2}$ are $Z_{p} G$-projective, since they are induced from groups of order prime to $p$, hence $X_{p} \cong\left(B \oplus U_{n}\right)_{p} \cong\left(V_{n} \oplus Z\right)_{p}$. So $B_{p}$ is $Z_{p} G$-invertible, and hence $Z_{p} H$-invertible. So $B$ is $Z G$-invertible. This implies by e.g. [B, Lemma 1.2]

$$
B \oplus U_{n} \cong V_{n} \oplus Z
$$

So $B$ is stably permutation.

Lemma 1.2. Let $X=Z / p Z$ be the trivial $Z G$-module with $p$ elements. Then there exists a $Z G$-exact sequence

$$
0 \rightarrow A \rightarrow A^{*} \rightarrow X \rightarrow 0
$$


Proof. $U_{n}^{*}$ is generated by $u_{i}^{*}$, where $u_{i}^{*}\left(u_{j}\right)=\delta_{i j}$, and $A^{*}$ is generated by $\operatorname{res}\left(u_{i}^{*}\right) I=$ $1, \ldots, n-1$, where res is restriction from $U_{n}$ to $A$. The map $A^{*} \rightarrow X$ is given by $\operatorname{res}\left(u_{i}^{*}\right) \rightarrow 1$.

Remark. Tensoring the sequence of Lemma 1.2 by $A$ we get

$$
0 \rightarrow A \otimes A \rightarrow A^{*} \otimes A \rightarrow X \otimes A \rightarrow 0 .
$$

Identifying $A \otimes A$ with $G_{n}$, and setting $T=X \otimes A \cong A / p A$ we obtain

$$
0 \rightarrow G_{n} \rightarrow B \rightarrow T \rightarrow 0 .
$$

Let $U=U_{n} / p U_{n}$. It is easy checked that the map $U \rightarrow X$ given by $u_{i} \rightarrow 1$ has kernel $T$. Thus we have a $Z G$-exact sequence

$$
0 \rightarrow T \rightarrow U \rightarrow X \rightarrow 0 .
$$

Let $T^{\prime}=A^{*} / p A^{*}$, then it is immediate that the following sequence is $Z G$-exact

$$
0 \rightarrow X \rightarrow U \rightarrow T^{\prime} \rightarrow 0 .
$$

These are important sequences which will be used in the following sections.

2 .

Throughout the rest of this paper we adopt the following notation unless otherwise specified. We will also keep the notation used in section 1.

- $G=S_{n}, n=p$ is prime; $n$ and $p$ will be used interchangeably without mention.

- $H=p$-sylow subgroup of $G$, so $H$ is cyclic of order $p$.

- $N=N_{G}(H)$ will be the normalizer of $H$ in $G$, so $N=H \rtimes C$, where $C$ is cyclic of order $p-1$, and if we let $h$ and $c$ generate $H$ and $C$ respectively, then $c h c^{-1}=h^{a}$ where $a$ is a primitive $(p-1)$ st root of $1 \bmod p$.

- $F=Z / p Z$ field of $p$ elements.

- For any finite group $G$, and for any $Z G$-lattice $M$, we will denote by $\phi(M)$ the flasque class of $M$, see [CT, section 1] for definitions.

- $Z^{-}$will denote the sign representation of $G$.

- $X$ will denote the trivial $Z G$-module of $p$ elements.

Definitions. (1) A $Z G$-lattice $M$ is quasi-permutation [EM], if there exist an exact sequence

$$
0 \rightarrow M \rightarrow R \rightarrow Q \rightarrow 0
$$

with $R$ and $Q$ permutation.

(2) We will say that 2 fields are stably isomorphic as $G$-fields, if the fields are stably isomorphic, and the isomorphism respects their $G$-actions.

Various versions of the following lemma have been proved in the literature, we need a slightly different version here.

Lemma 2.1. Let $G$ be a finite group.

(1) Suppose there exists an exact sequence of $Z G$-lattices

$$
0 \rightarrow M \rightarrow N \rightarrow E \rightarrow 0
$$

with $E$ invertible and $M, G$-faithful, then $\mathbf{C}(E \oplus M) \cong \mathbf{C}(N)$ as $G$-fields. 
(2) Let $G$ be a finite group, and let $M$ and $M^{\prime}$ be $G$-faithful $Z G$-lattices, with $M$ quasi-permutation. Then the fields $\mathbf{C}\left(M \oplus M^{\prime}\right)$ and $\mathbf{C}\left(M^{\prime}\right)$ are stably isomorphic as G-fields.

(3) Let $M$ and $M^{\prime}$ be $G$-faithful $Z G$-lattices such that $\phi(M)=\phi\left(M^{\prime}\right)$, then $\mathbf{C}(M)$ and $\mathbf{C}\left(M^{\prime}\right)$ are stably isomorphic as $G$-fields.

(4) If $G=S_{n}$ and $M$ is a $G$-faithful quasi-permutation $Z G$-lattice, then $\mathbf{C}(M)^{G}$ is stably rational over $\mathbf{C}$.

Proof. (1) The proof is basically that of [L, Proposition 1.5]. The injection $M \rightarrow N$ induces an injection $\mathbf{C}(M) \subset \mathbf{C}(N)$. Now consider the sequence

$$
0 \rightarrow \mathbf{C}(M)^{*} \rightarrow \mathbf{C}(M)^{*} N \rightarrow E \rightarrow 0 .
$$

Since $G$ acts faithfully on $\mathbf{C}(M)^{*}, \mathbf{C}(M)^{*}$ is $H^{1}$-trivial by Hilbert theorem 90 . Since $E$ is invertible the sequence splits by [CT, Lemma 1, section 1]. Thus $\mathbf{C}(M)^{*} N \cong$ $E \oplus \mathbf{C}(M)^{*}$. This isomorphism yields the required isomorphism of $G$-fields.

(2) Since $M$ is quasi-permutation there exist an exact sequence

$$
0 \rightarrow M \rightarrow R \rightarrow Q \rightarrow 0
$$

with $Q$ and $R$ permutation. By (1) $\mathbf{C}(M \oplus Q) \cong \mathbf{C}(R)$. So $\mathbf{C}\left(M \oplus M^{\prime} \oplus Q\right) \cong$ $\mathbf{C}\left(M^{\prime} \oplus R\right)$. Apply [L, Proposition 1.3] to the field $l=\mathbf{C}\left(M^{\prime}\right)$ and the $l$-vector space $W=\sum l r_{i}$, where $\left\{r_{i}\right\}$ is a $Z$-basis for $R$ which is permuted by $G$. Then there exists elements $\left\{y_{i}\right\}$ in $W^{G}$ which form an $L$-basis for $W$. Now $W \subset l(R)$, and hence

$$
l(R)=l\left(y_{1}, \ldots, y_{k}\right)=\mathbf{C}\left(M^{\prime}\right)\left(y_{1}, \ldots, y_{k}\right) .
$$

By the same argument there exist indeterminates $z_{1}, \ldots, z_{t}$, such that

$$
\mathbf{C}\left(M \oplus M^{\prime} \oplus Q\right)=\mathbf{C}\left(M \oplus M^{\prime}\right)\left(z_{1}, \ldots, z_{t}\right) .
$$

So $\mathbf{C}\left(M^{\prime}\right)\left(y_{1}, \ldots, y_{k}\right) \cong \mathbf{C}\left(M \oplus M^{\prime}\right)\left(z_{1}, \ldots, z_{t}\right)$.

(3) Since $\phi(M)=\phi\left(M^{\prime}\right)$, there exist 2 exact sequences

$$
0 \rightarrow M \rightarrow E \rightarrow R \rightarrow 0 \text { and } 0 \rightarrow M^{\prime} \rightarrow E \rightarrow P \rightarrow 0
$$

with $P$ and $R$ permutation by [CT, Lemma 8 , section 1]. By $(1) \mathbf{C}(E) \cong$ $\mathbf{C}(M \oplus R) \cong \mathbf{C}\left(M^{\prime} \oplus P\right)$ as $G$-fields, and by (2) $\mathbf{C}(M)$ ad $\mathbf{C}\left(M^{\prime}\right)$ are stably isomorphic as $G$-fields.

(4) Since $M$ is quasi-permutation there is an exact sequence

$$
0 \rightarrow M \rightarrow R \rightarrow Q \rightarrow 0
$$

with $Q$ and $R$ permutation. By (1) $\mathbf{C}(M \oplus Q) \cong \mathbf{C}(R)$. By (2) $\mathbf{C}(M \oplus Q)$ and $\mathbf{C}(M)$ are stably isomorphic as $G$-fields. Since $\phi\left(U_{n}\right)=\phi(R)=0, \mathbf{C}\left(U_{n}\right)$ and $\mathbf{C}(R)$ stably isomorphic as $G$-fields by (3). Since $\mathbf{C}\left(U_{n}\right)^{G}$ is rational over $\mathbf{C}$, the result follows.

Proposition 2.2. (1) Let $G$ be a finite group and suppose there exist exact sequences

$$
0 \rightarrow E \rightarrow P \rightarrow L \rightarrow 0 \text { and } 0 \rightarrow E^{\prime} \rightarrow R \rightarrow J \oplus L \rightarrow 0
$$

with $P$ and $R, Z G$-permutation, $E$ and $E^{\prime} G$-faithful, $L$ and $J$, finite and $J=$ $Q / m Q$ for some $Z G$-permutation module $Q$, and some positive integer $m$. Then the fields $\mathbf{C}\left(E^{\prime}\right)$ and $\mathbf{C}(E)$ are stably isomorphic as $G$-fields. 
(2) Let $G=S_{n}$ and let $M$ be a $G$-faithful $Z G$-lattice of trivial cohomology, then $\mathbf{C}(M)^{G}$ is stably rational over $\mathbf{C}$. Furthermore if $M^{\prime}$ is any $G$-faithful ZG-lattice, then the fields $\mathbf{C}\left(M \oplus M^{\prime}\right)$ and $\mathbf{C}\left(M^{\prime}\right)$ are stably isomorphic as $G$-fields.

Proof. (1) We form the commutative diagram

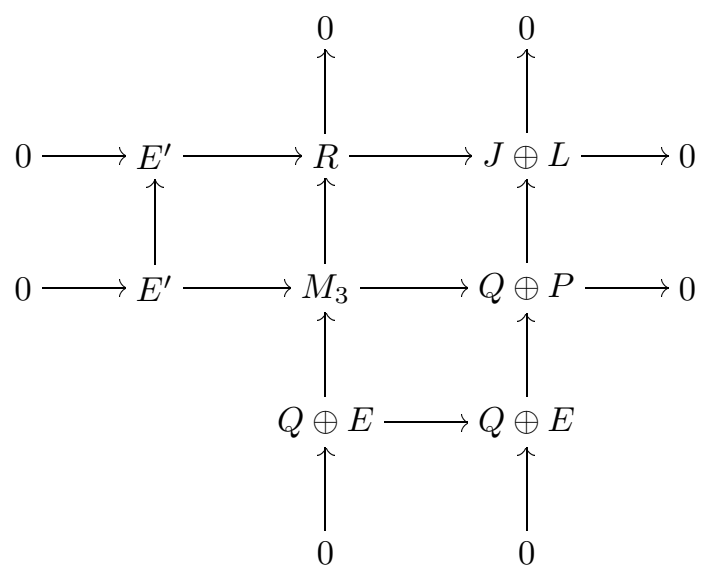

Since $R, Q$ and $P$ are permutation modules, the fields $\mathbf{C}(E)$ and $\mathbf{C}\left(E^{\prime}\right)$ are stably isomorphic as $G$-fields, by Lemma 2.1 .

(2) By [BK, Theorem 8.10] $M$ is $Z G$-projective and by [EM, Theorem 3.3] $M$ is stably permutation. The lemma follows by Lemma 2.1 .

Notation. Let $I_{G / N}$ denote the kernel of the augmentation map $Z(G / N) \rightarrow Z$, and let $I=I_{G / N} / p I_{G / N}$.

Proposition 2.3. The $Z_{p} G$-lattice $\left(I_{G / N}\right)_{p}$ and the $Z G$-lattice $I$ are cohomologically trivial. Let $I_{G / N}^{-}=I_{G / N} \otimes Z^{-}, I^{-}=I \otimes Z^{-}$, then $\left(I_{G / N}^{-}\right), I^{-}$are also cohomologically trivial.

Proof. The sequence $0 \rightarrow\left(I_{G / N}\right)_{p} \rightarrow Z_{p}(G / N) \rightarrow Z_{p} \rightarrow 0$ splits with splitting map $Z_{p} \rightarrow Z_{p}(G / N)$ given by $1 \rightarrow\left(\sum_{g \in G / N} g\right) /[G: N]$. So $Z_{p}(G / N) \cong Z_{p} \oplus\left(I_{G / N}\right)_{p}$. By Mackey's subgroup theorem [CR1, Theorem 10.13] $\operatorname{Res}_{H}^{G} Z G / N \cong Z \oplus Z H^{k}$ for some positive integer $k$. So as a $\widehat{Z}_{p} H$-module $\left(\hat{I}_{G / N}\right)_{p}$ is free by the Krull-SchmidtAzumaya and by [CR1, Theorem 30.17] $\left(I_{G / N}\right)_{p}$ is a free $Z_{p} H$-module. Now for any subgroup $K$ of $G, H^{i}\left(K,\left(I_{G / N}\right)_{p}\right)$ injects into $H^{i}\left(H,\left(I_{G / N}\right)_{p}\right)=0$. This proves that $\left(I_{G / N}\right)_{p}$ is cohomologically trivial. To prove that $I$ is cohomologically trivial it suffices to take the cohomology of the sequence

$$
0 \rightarrow\left(I_{G / N}\right)_{p} \rightarrow\left(I_{G / N}\right)_{p} \rightarrow I \rightarrow 0 .
$$

The second statement follows since $I_{G / N}^{-}, I^{-}$are isomorphic to $I_{G / N}$ and $I$ respectively as $H$-modules.

As in the preceding proof we have $F G / N \cong F \oplus I$. The following proposition is a crucial reduction step. In particular it implies Theorem 2.6 which says that $\mathbf{C}\left(G_{n}\right)$ and $\mathbf{C}\left(Z G \otimes_{Z N} G_{n}\right)$ are stably isomorphic as $G$-fields, so that we can basically study $G_{n}$ at the $N$-level and induce up.

Proposition 2.4. Let $L$ be a finite $Z G$-mdoule of exponent $p$. Suppose we have 
(1) An FG-extension of $X$ or $X^{-}$by $L$, or of $L$ by $X$ or $X^{-}$, whose middle term is FG-projective.

(2) A ZG-exact sequence

$$
0 \rightarrow M \rightarrow P \rightarrow L \oplus Q / m Q \rightarrow 0
$$

with $P$ and $Q$ stably permutation, where $m$ is a positive integer.

Then the fields $\mathbf{C}(M)$ and $\mathbf{C}\left(Z G \otimes_{Z N} M\right)$ are stably isomorphic as $G$-fields.

Proof. Tensoring the sequence

$$
0 \rightarrow M \rightarrow P \rightarrow L \oplus Q / m Q \rightarrow 0
$$

by $Z G$ over $Z N$ we get

$$
0 \rightarrow Z G \otimes_{Z N} M \rightarrow Z G \otimes_{Z N} P \rightarrow F G \otimes_{F N} L \oplus Z G \otimes_{Z N} Q / m Q \rightarrow 0
$$

We form the sequence

$$
0 \rightarrow Q \rightarrow Q \rightarrow Q / m Q \rightarrow 0
$$

and add it to (s2) to obtain

$$
\begin{aligned}
0 & \rightarrow Z G \otimes_{Z N} M \oplus Q \rightarrow Z G \otimes_{Z N} P \oplus Q \\
& \rightarrow F G \otimes_{F N} L \oplus Z G \otimes_{Z N} Q / m Q \oplus Q / m Q \rightarrow 0 .
\end{aligned}
$$

We have $F G \otimes_{F N} L \cong L \oplus I \otimes L$, and we assume that we have an $F G$-exact sequence of the form

$$
0 \rightarrow L \rightarrow U \rightarrow X \rightarrow 0
$$

The proof extends directly to the other cases. Tensoring (1) by $I$ of $F$ preserves $F G$-exactness since $I$ is $F$-free, and we get

$$
0 \rightarrow L \otimes I \rightarrow U \otimes I \rightarrow I \rightarrow 0
$$

Since $U$ is $F G$-projective, so is $U \otimes I$. By looking at the cohomology of the $Z G$-exact sequence

$$
0 \rightarrow Z G \rightarrow Z G \rightarrow F G \rightarrow 0,
$$

we see that $F G$ is cohomologically trivial as a $Z G$-module, therefore so is $U \otimes I$. By Proposition 2.3 so is $I$. Thus $L \otimes I$ is also $Z G$-cohomologically trivial. Now consider the exact sequence

$$
0 \rightarrow M^{\prime} \rightarrow M^{\prime \prime} \rightarrow L \otimes I \rightarrow 0 \text { with } M^{\prime \prime} \text { free. }
$$

Since $M^{\prime \prime}$ and $L \otimes I$ are cohomologically trivial so is $M^{\prime}$, thus $M^{\prime}$ is $Z G$-projective by [BK, Theorem 8.10], and by [EM, Theorem 3.3] it is stably permutation. Now add the sequences (s1), (s5) and

$$
0 \rightarrow Z G \otimes_{Z N} Q \rightarrow Z G \otimes_{Z N} Q \rightarrow Z G \otimes_{Z N} Q / m Q \rightarrow 0
$$


and form a commutative diagram with (s4),

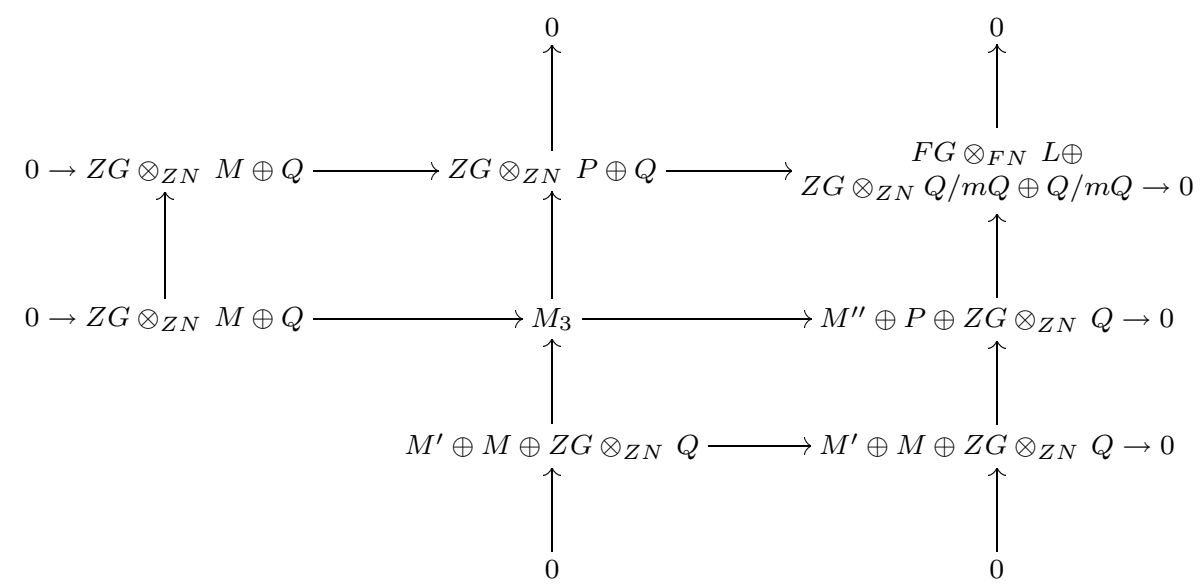

The result now follows by Lemma 2.1, since $M^{\prime \prime}, P, M^{\prime}, Q$ and $Z G \otimes_{Z N} Q$ are stably permutation for this case. A similar argument applies to the other cases.

Corollary 2.5. The fields $\mathbf{C}\left(A^{*}\right)$ and $\mathbf{C}\left(Z G \otimes_{Z N} A^{*}\right)$ are stably isomorphic as $G$-fields. So are the fields $\mathbf{C}\left(A^{*-}\right)$ and $\mathbf{C}\left(Z G \otimes_{Z N} A^{*-}\right)$.

Proof. To prove the first statement of the corollary, we note that there exists an exact sequence

$$
0 \rightarrow A \oplus Z \rightarrow U_{n} \rightarrow X \rightarrow 0
$$

where the map from $U_{n}$ to $X$ is given by $u_{i} \rightarrow 1$. Tensoring the sequence by $A^{*}$ over $Z$ we get

$$
0 \rightarrow B \oplus A^{*} \rightarrow V \rightarrow T^{\prime} \rightarrow 0,
$$

where $T^{\prime}=A^{*} / p A^{*}$ as in section 1 .

By the remark at the end of section 1 we have an exact sequence

$$
0 \rightarrow X \rightarrow U \rightarrow T^{\prime} \rightarrow 0 .
$$

Hence by Proposition 2.4, $\mathbf{C}\left(A^{*} \oplus B\right)$ and $\mathbf{C}\left(Z G \otimes_{Z N}\left(A^{*} \oplus B\right)\right)$ are stably isomorphic as $G$-fields. The result follows by Lemma 2.1 since $B$ is stably permutation. For the second statement we have the exact sequence

(1) $0 \rightarrow A^{*-} \oplus Z^{-} \rightarrow U_{n}^{-} \rightarrow T^{\prime-} \rightarrow 0$, where the map $U_{n}^{-} \rightarrow T^{-} \rightarrow 0$ is given by $U_{n} \rightarrow A^{*} \rightarrow T^{\prime} \rightarrow 0$. We also have the sequence $0 \rightarrow Z \oplus Z^{-} \rightarrow Z G / A_{n} \rightarrow J \rightarrow 0$ where $J=Z / 2 Z$.

Tensoring by $U_{n}$ we get

(2) $0 \rightarrow U_{n}^{-} \oplus U_{n} \rightarrow Z G \otimes_{Z A_{n}} U_{n} \rightarrow J^{\prime} \rightarrow 0$ where $J^{\prime}=U_{n} / 2 U_{n}$, and $A_{n}$ is the alternating group.

Adding $U_{n}$ to the first 2 terms of (1) and combining with (2) we get

$$
0 \rightarrow A^{*-} \oplus Z^{-} \oplus U_{n} \rightarrow Z G \otimes Z A_{n} U_{n} \rightarrow T^{\prime-} \oplus J^{\prime} \rightarrow 0
$$

since $T^{\prime-}$ and $J^{\prime}$ are of relatively prime orders.

So $\mathbf{C}\left(A^{*-} \oplus Z^{-} \oplus U_{n}\right)$ and $\mathbf{C}\left(Z G \otimes_{Z N}\left(A^{*-} \oplus Z^{-} \oplus U_{n}\right)\right)$ are stably isomorphic as $G$-fields, by Proposition 2.4. Now the result follows by Lemma 2.1 and by observing that $Z^{-}$is quasi-permutation. 
The main theorem is a direct consequence of Proposition 2.4.

Theorem 2.6. The fields $\mathbf{C}\left(G_{n}\right)$ and $\mathbf{C}\left(Z G \otimes_{Z N} G_{n}\right)$ are stably isomorphic as $G$-fields. The center $C_{n}$ is isomorphic to their fixed fields.

Proof. By the remark at the end of section 1, there is an exact sequence

$$
0 \rightarrow T \rightarrow U \rightarrow X \rightarrow 0 .
$$

Applying Proposition 2.4 to the $Z G$-exact sequence: $0 \rightarrow G_{n} \rightarrow B \rightarrow T \rightarrow 0$ from section 1 , we get that $\mathbf{C}\left(G_{n}\right)$ and $\mathbf{C}\left(Z G \otimes_{Z N} G_{n}\right)$ are stably isomorphic as $G$ fields. By [F1, Theorem 3] and [L, Proposition 1.4] we have $C_{n}$ ad $\mathbf{C}\left(G_{n}\right)^{G}$ stably isomorphic.

Theorem 2.7. Let $D$ and $R$ be invertible $Z N$-lattices. Suppose that $R$ is either 0 , or stably permutation and $Z_{q} C$-projective for all primes $q$ dividing $p-1$. If $\left(G_{n} \oplus R\right)_{p} \cong D_{p}$, then the fields $\mathbf{C}\left(G_{n}\right)$ and $\mathbf{C}\left(Z G \otimes_{Z N} D\right)$ are stably isomorphic as $G$-fields.

Proof. We have $\left(G_{n} \oplus R\right)_{p} \cong D_{p}$. By [CR1, Lemma 31.4], this implies the existence of a $Z N$-exact sequence

$$
0 \rightarrow G_{n} \oplus R \rightarrow D \rightarrow Y \rightarrow 0
$$

where $Y$ is a finite $Z N$-module of order prime to $p$. Since $\left(G_{n} \oplus R\right)_{p} \cong D_{p}$, $Q\left(G_{n} \oplus R\right) \cong Q D$ where $Q$ is the field of rational numbers. It follows directly form the definition of $G_{n}$, that this lattice is $Z C$-free. Therefore $\left(G_{n} \oplus R\right)_{q}$ is $Z_{q} C$-projective for all primes $q$ dividing $p-1$, and [B1, Theorem 1.2] implies that so is $D_{q}$. Since the first 2 terms of the sequence are $Z_{q} C$-projective for all primes $q \neq p, Y$ is cohomologically trivial. Therefore we have a $Z N$-exact sequence

$$
0 \rightarrow \mathrm{Pr} \rightarrow \mathrm{Fr} \rightarrow Y \rightarrow 0
$$

with $\mathrm{Fr}$, free. Since $Y$ is cohomologically trivial, so is $\operatorname{Pr}$, hence $\operatorname{Pr}$ is $Z N$ projective by [BK, Theorem 8.10].

Forming a commutative diagram with the above sequences we obtain

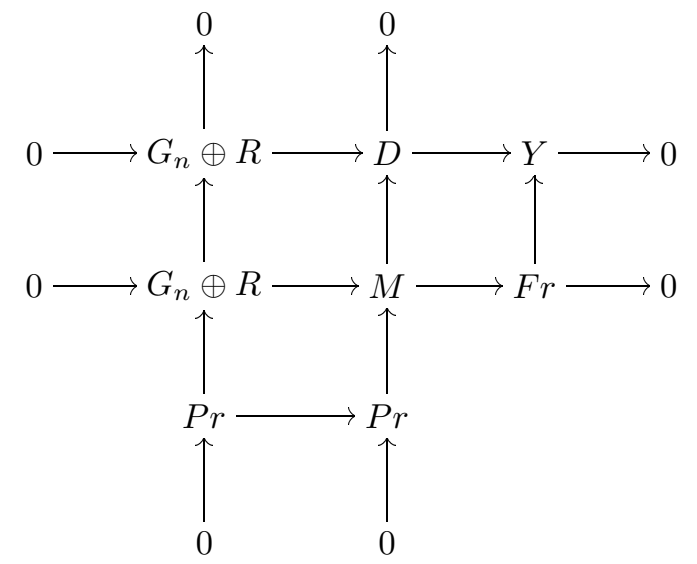

This implies that $G_{n} \oplus R \oplus F r \cong D \oplus \operatorname{Pr}$ since $P r$ and $F r$ are projective. Inducing up to $G$ we obtain

$$
Z G \otimes_{Z N}\left(G_{n} \oplus R \oplus F r\right) \cong Z G \otimes_{Z N}(D \oplus P r) .
$$


Since $\operatorname{Pr}$ and $\mathrm{Fr}$ are $Z N$-projective, the induced modules are $Z G$-projective and by [EM, Theorem 3.3] they are stably permutation. The result now follows by Lemma 2.1 and Theorem 2.6.

The following lemma will be needed later.

Lemma 2.8. There exist a $Z G$-lattice $W$ such that $G_{n} \oplus W \cong V_{n} \oplus Z(G / H)$. As $Z N$-lattices we have $G_{n} \oplus W \cong Z N^{k} \oplus Z(N / H)$ for some positive integer $k$.

Proof. Since $H$ is cyclic and $A \cong Z H(h-1)$ as a $Z H$-module, we have the $Z H$-exact sequence

$$
0 \rightarrow Z \rightarrow Z H \rightarrow A \rightarrow 0
$$

tensoring by $Z G$ over $Z H$ we get

$$
0 \rightarrow Z(G / H) \rightarrow Z G \rightarrow Z(G / H) \otimes A \rightarrow 0 .
$$

From this sequence we form the following diagram

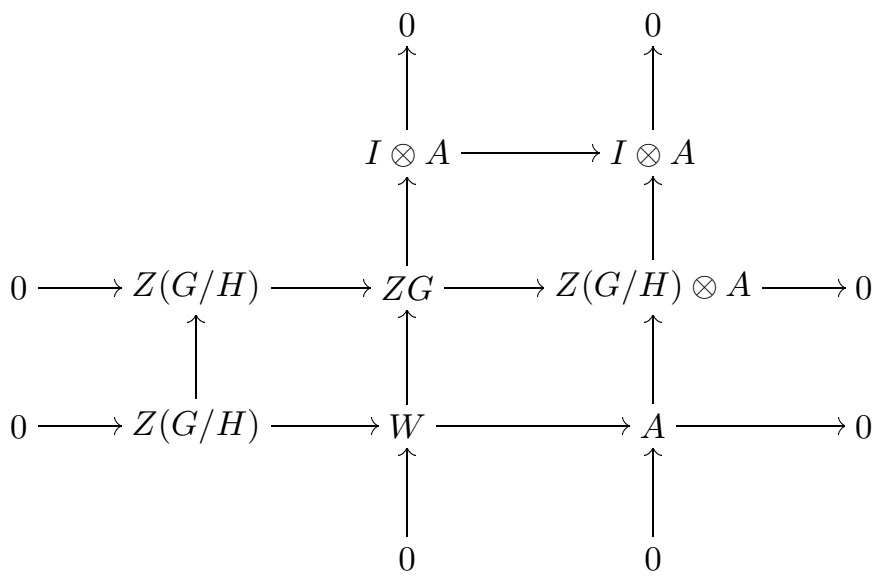

where $I=I_{G / H}$.

For all primes $q$ different from $p$, dividing the order of $G, W_{q} \cong(A \oplus Z(G / H))_{q}$, since $A_{q}$ is $Z_{q} G$-invertible by the proof of Proposition 1.1. For the prime $p$, we have

$$
Z_{p}(G / H) \cong Z_{p} \oplus I_{p} .
$$

So

$$
Z_{p}(G / H) \otimes A_{p} \cong A_{p} \oplus I_{p} \otimes A_{p} .
$$

Since $H^{0}(H, Z G / H \otimes A) \cong H^{1}(H, Z G / H)=0$, we have

$$
H^{1}(H, W) \cong H^{0}(H, I \otimes A)=0,
$$

and thus $W_{p}$ is $Z_{p} H$-invertible since $H$ is cyclic by [CT, Corollary 2 and Proposition 2, section 1]. By [B, Theorem 2.1], $W$ is invertible. We have the following 
commutative diagram

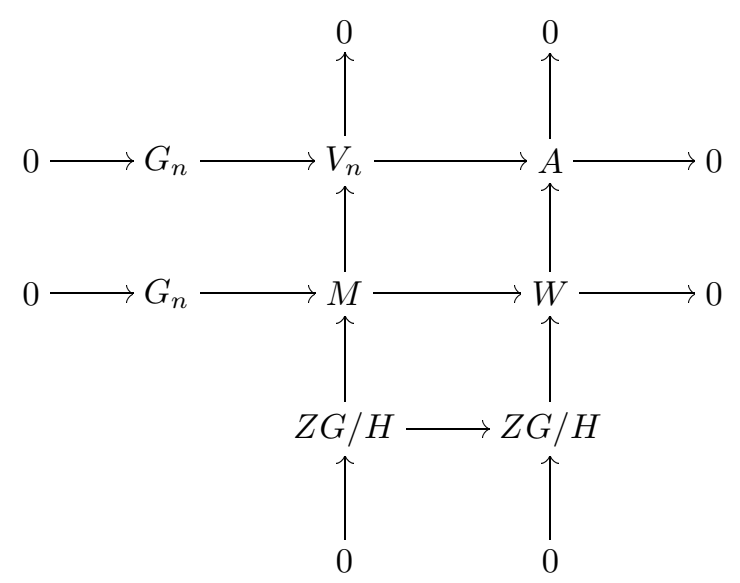

Since $V_{n}, G_{n}, W$ and $Z G / H$ are invertible, $V_{n} \oplus Z G / H \cong W \oplus G_{n}$ by [CT, Lemma 1 , section 1]. The second statement follows from the fact that $V_{n} \cong Z N$ and $Z(G / H) \cong Z(N / H) \oplus Z N^{k-1}$ as $Z N$-lattices.

3.

Recall that $N$ was defined to be the normalizer of a $p$-sylow subgroup $H$ of $G$. So $N=N_{G}(H)=H \rtimes C$, where $C$ is cyclic of order $p-1$, and if we let $h$ and $c$, generate $H$ and $C$ respectively than $c . h=h^{a}$, where $a$ is primitive $(p-1)$ st root of $1 \bmod p$.

Now

$$
\widehat{Z}_{p} N / H=\bigoplus_{k=1}^{p-1} Z_{k}
$$

where $Z_{k}$ is the $\widehat{Z}_{p} N$-module of rank 1 with trivial $H$-action, and such that $c$ acts on 1 as $\theta^{k}$, where $\theta$ was chosen to be the primitive $(p-1)$-st root of 1 in $\widehat{Z}_{p}$ for which $\theta \equiv a \bmod p$.

We let $X_{k}=Z_{k} / p Z_{k}$ for $k=1, \ldots, p-1$. So $X=X_{p-1}$ is the trivial $Z_{p} N$-module $Z / p Z$.

Lemma 3.1. Keeping the definitions of $A$ and $A^{*}$. The $Z_{p} N$-modules $A_{p}$ and $A_{p}^{*}$ are isomorphic as $Z_{p} H$-modules. Therefore $Z_{p} N \otimes Z_{p} H A \cong Z_{p} N \otimes_{Z_{p} H} A^{*}$.

Proof. As a $Z N$-module $U_{n} \cong Z N / C \cong Z H$ where $u_{i} \rightarrow h^{i}$ and $c . h=h^{a}$. Thus $A \cong Z H(h-1)$. Since $H$ is cyclic we have a $Z H$-exact sequence

$$
0 \rightarrow Z \rightarrow Z H \rightarrow Z H(h-1) \rightarrow 0 .
$$

Equivalently

$$
0 \rightarrow Z \rightarrow Z H \rightarrow A \rightarrow 0
$$

From this sequence and the sequence

$$
0 \rightarrow Z \rightarrow Z H \rightarrow A^{*} \rightarrow 0 .
$$


We obtain the commutative diagram of $Z H$-modules.

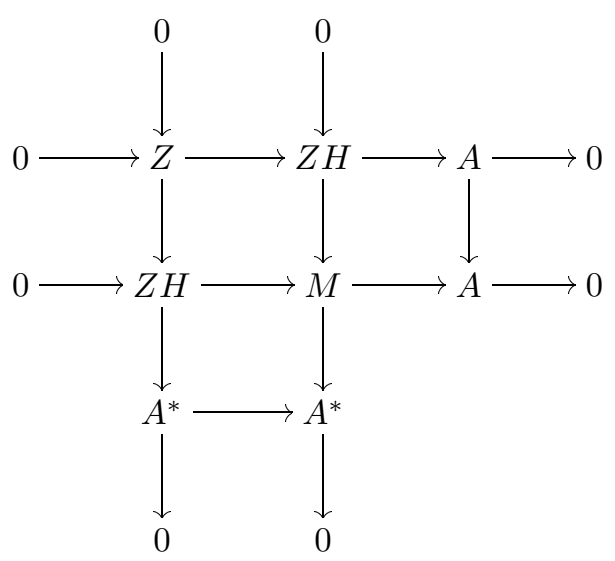

Thus $A^{*} \oplus Z H \cong A \oplus Z H$. By the Krull-Schmidt-Azumaya (K-S-A) $\widehat{A}_{p} \cong \widehat{A}_{p}^{*}$, and by [CR1, Theorem 30.17] $A_{p}$ and $A_{p}^{*}$.

Notation. For each $m$ dividing $p-1$, we denote by $C_{m}$ the subgroup of $C$ generated by $c^{m}$, thus $C_{m}$ has order $(p-1) / m$, and index $m$ in $C$.

We will denote by $A_{k}$ the $\widehat{Z}_{p} N$-lattice $\widehat{A}_{p}^{*} \otimes Z_{k}$, for $k=1, \ldots, p-1$.

For each $k$, we will denote by $G_{k}$ the $\widehat{Z}_{p} N$-lattice $\widehat{A}_{p}^{*} \otimes A_{k}$.

Theorem 3.2. We have the following isomorphisms of $\widehat{Z}_{p} N$-lattices.

(1) $A^{*} \otimes Z_{1} \cong \widehat{A}_{p}$ as $Z_{p} N$-lattices.

(2) $\left(\widehat{G}_{N}\right)_{p} \cong \widehat{A}_{p}^{*} \otimes A_{2} \cong G_{2}$.

(3) $\widehat{B}_{p} \cong G_{1}$.

Proof. By Lemma 3.1 we have $Z_{p} N \otimes_{Z_{p} H} A \cong Z_{p} N \otimes_{Z_{p} H} A^{*}$. Thus

$$
\bigoplus_{k=1}^{p-1} A_{k} \cong \bigoplus_{k-1}^{p-1} \widehat{A}_{P} \otimes Z_{k} .
$$

As an $Z_{p} H$-module $A_{p}^{*} \cong Z_{p}[w]$ where $w$ is a primitive $p$-th root of 1 on $Z_{p}$. Therefore $\widehat{A}_{p}^{*}$ is indecomposable as a $\widehat{Z}_{p} H$-module and a fortiori as a $Z_{p} N$-module. By the K-S-A, this implies that $\widehat{A}_{p} \cong \widehat{A}_{p}^{*} \otimes Z_{j}$ for some $j$. We will show that $j=1$. Since $\operatorname{Ext}_{\widehat{Z}_{p} N}^{1}\left(\widehat{A}_{p}, \widehat{Z}_{p} N / H\right) \cong \operatorname{Ext}_{\widehat{Z}_{p} N}^{1}\left(\widehat{Z}_{p} N / H, A_{p}^{*}\right) \cong H^{1}\left(H, A_{p}^{*}\right) \cong Z / p$ we have $\operatorname{Ext}_{\widehat{Z}_{p} N}^{1}\left(\widehat{A}_{p}, Z_{k}\right)=0$ for all $k$ except for a unique $k$ for which $\operatorname{Ext}_{\widehat{Z}_{p} N}^{1}\left(\widehat{A}_{p}, Z_{k}\right)=Z / p$. It is easily seen that $Z_{k}^{*}=\operatorname{Hom}\left(Z_{k}, Z_{p}\right) \cong Z_{p-1-k}$, for $k=1, \ldots, p-1$.

Now taking the cohomology of the sequence

$$
0 \rightarrow \widehat{A}_{p} \rightarrow\left(\widehat{U}_{n}\right)_{p} \rightarrow \widehat{Z}_{p} \rightarrow 0
$$

we have $0 \rightarrow \operatorname{Hom}_{N}\left(\widehat{Z}_{p}, Z_{k}\right) \rightarrow \operatorname{Hom}_{N}\left(\left(\widehat{U}_{n}\right)_{p}, Z_{k}\right) \rightarrow \operatorname{Hom}_{N}\left(\widehat{A}_{p}, Z_{k}\right) \rightarrow$ $\operatorname{Ext}_{N}^{1}\left(\widehat{Z}_{p}, Z_{k}\right)=0$ since $Z_{k}$ is invertible. Now if $k \neq p-1$ then $\operatorname{Hom}_{N}\left(\widehat{Z}_{p}, Z_{k}\right) \cong$ $Z_{k}^{N}=0$, and $\operatorname{Hom}_{N}\left(\left(\widehat{U}_{n}\right)_{p}, Z_{k}\right) \cong Z_{k}^{C} \cong 0$, thus $\operatorname{Hom}_{N}\left(\widehat{A}_{p}, Z_{k}\right) \cong 0$. If $k=p-1$, then $\operatorname{Hom}_{N}\left(\widehat{Z}_{p}, Z_{k}\right) \cong \widehat{Z}_{p}, \operatorname{Hom}_{N}\left(\left(\widehat{U}_{n}\right)_{p}, Z_{k}\right) \cong \widehat{Z}_{p}$. Since $\operatorname{Hom}_{N}\left(\widehat{A}_{p}, Z_{k}\right)$ is torsion free it must be 0 . 
Now consider the exact sequence

$$
0 \rightarrow Z_{k} \rightarrow Z_{k} \rightarrow X_{k} \rightarrow 0 .
$$

From this we have $0 \rightarrow \operatorname{Hom}_{N}\left(\widehat{A}_{p}, Z_{k}\right) \rightarrow \operatorname{Hom}_{N}\left(\widehat{A}_{p}, Z_{k}\right) \rightarrow \operatorname{Hom}_{N}\left(\widehat{A}_{p}, X_{k}\right) \rightarrow$ $\operatorname{Ext}_{N}^{1}\left(\widehat{A}_{p}, Z_{k}\right) \rightarrow 0$. Therefore the unique $k$ for which $\operatorname{Ext}_{N}^{1}\left(\widehat{A}_{p}, Z_{k}\right) \cong Z / p$ is the $k$ for which $\operatorname{Hom}_{N}\left(A_{p}, X_{k}\right)=Z / p$. Now we have a nonzero $Z_{p} N$-homomorphism $A_{p} \rightarrow X_{1}$ given by $h-1 \rightarrow 1$. So $k=1$.

Now tensoring the sequence

$$
0 \rightarrow \widehat{Z}_{p} \rightarrow\left(\widehat{U}_{n}\right)_{p} \rightarrow A_{p}^{*} \rightarrow 0
$$

by $Z_{j}$, and using the fact that $\widehat{A}_{p} \cong \widehat{A}_{p}^{*} \otimes Z_{j}$ we obtain

$$
0 \rightarrow Z_{j} \rightarrow\left(\widehat{U}_{n}\right)_{p} \otimes Z_{j} \rightarrow \widehat{A}_{p} \rightarrow 0
$$

This sequence is not split, hence $j=1$. This proves $(1)$.

To prove (2), we tensor both sides of $\widehat{A}_{p} \cong \widehat{A}_{p}^{*} \otimes Z_{1}$ by $\widehat{A}_{p}$ and we get

$$
\left(\widehat{G}_{n}\right)_{p} \cong \widehat{A}_{p}^{*} \otimes Z_{1} \otimes \widehat{A}_{p}^{*} \otimes Z_{1} \cong \widehat{A}_{p}^{*} \otimes \widehat{A}_{p}^{*} \otimes Z_{2} \cong G_{2}
$$

To prove (3), we tensor $\widehat{A}_{p} \cong \widehat{A}_{p}^{*} \otimes Z_{1}$ by $A_{p}^{*}$ and we get

$$
B_{p} \cong A_{p}^{*} \otimes \widehat{A}_{p} \cong \widehat{A}_{p}^{*} \otimes \widehat{A}_{p}^{*} \otimes Z_{1} \cong G_{1} .
$$

Remark. The lattice $A_{2}$ is an important lattice for the following reason. Consider the exact $Z_{p} N$-sequence

$$
0 \rightarrow \widehat{Z}_{p} \rightarrow \widehat{Z}_{p} H \rightarrow A_{p}^{*} \rightarrow 0
$$

Tensoring by $A_{2}$ we get

$$
0 \rightarrow A_{2} \rightarrow \widehat{Z}_{p} H \otimes A_{2} \rightarrow G_{2} \rightarrow 0
$$

Since $Z H \cong Z(N / C), \operatorname{Res}_{C}^{N} U_{n} \cong Z \oplus Z C$ by Mackey's subgroup theorem [CR1, Theorem 10.13]. As in the proof of Proposition 1.1, $\left(\widehat{U}_{n}\right)_{q} \cong A_{q}^{*} \oplus Z_{q} \cong A_{q} \oplus Z_{q}$ for all primes $q$ dividing $p-1$. By the K-S-A and by [CR1, Theorem 30.17] this implies that $A_{q}^{*} \cong A_{q} \cong Z_{q} C$. If we let $Q$ be the rationals this implies that $Q A \cong Q A^{*}$ as $Q C$-modules. Since $Z_{p} C$ is a maximal order in $Q C$ we have $A_{p} \cong A_{p}^{*} \cong Z_{p} C$ as $Z_{p} C$-modules by [CR1, Proposition 31.2]. Thus $A_{k} \cong \widehat{Z}_{p} C$ as $\widehat{Z}_{p} C$-modules for all $k=1, \ldots, p-1$. Hence $\widehat{Z}_{p} H \otimes A_{2} \cong \widehat{Z}_{p} N$, so $\phi\left(A_{2}\right)=\left[G_{2}\right]$. By the same argument we have $\phi\left(A_{k}\right)=\left[G_{k}\right]$ for all $k=1, \ldots, p-1$.

By the above $\left(G_{n}\right)_{q},\left(G_{n}^{*}\right)_{q}$ and $B_{q}$ are $Z_{q} C$-free for all primes $q$ dividing $p-1$. This fact will be needed later.

We will now use the machinery developed here to give new and very simple proofs for $p=5$ and $p=7$.

Theorem 3.3. The center of the ring of $5 \times 5$ generic matrices is stably rational over $C$.

Proof. Let $p=5$; then

$$
\widehat{Z}_{p} N / H=\bigoplus_{k=1}^{4} Z_{k}
$$

and so

$$
\widehat{Z}_{p} N \otimes_{\widehat{Z}_{p} H} A_{p}^{*}=A_{p}^{*} \oplus A_{1} \oplus A_{2} \oplus A_{3}
$$


Now $A_{2} \cong \widehat{A}_{p}^{*} \otimes Z_{2} \cong \widehat{A}_{p}^{*-}$, since $Z_{2} \cong \widehat{Z}_{p}^{-}$. Tensoring both sides of $A_{2} \cong \widehat{A}_{p}^{*} \otimes Z_{2}$ by $\widehat{A}_{p}^{*}$ we get by Theorem 3.2

$$
\left(\widehat{G}_{N}\right)_{p} \cong\left(\widehat{G}_{N}^{*-}\right)_{p}
$$

Now

$$
\widehat{Z}_{p} N \otimes_{Z_{p} H C_{2}} A_{p}^{*} \cong \widehat{A}_{p}^{*} \oplus A_{2}
$$

Tensoring for $\widehat{A}_{p}^{*}$ we get

$$
\widehat{Z}_{p} N \otimes Z_{p} H C_{2}\left(\widehat{G}_{N}^{*}\right)_{p} \cong\left(\widehat{G}_{N}^{*}\right)_{p} \oplus\left(\widehat{G}_{N}^{*-}\right)_{p} .
$$

Therefore

$$
Z_{p} N \otimes_{Z_{p} H C_{2}}\left(G_{n}^{*}\right)_{p} \cong\left(G_{n}^{*}\right)_{p} \oplus\left(G_{n}\right)_{p},
$$

and by [CR1, Theorem 30.17]

$$
Z_{p} N \otimes_{Z_{p} H C_{2}}\left(G_{n}^{*}\right)_{p} \cong\left(G_{n}^{*}\right)_{p} \oplus\left(G_{n}\right)_{p} .
$$

By [CT, Proposition 3, section 1], $\phi\left(\left(A_{n}^{*}\right)\right)=0$ as a $Z H C_{2}$-module since $C_{2}$ is of order 2. Since $G_{n}^{*}$ is invertible, this implies that $Z N \otimes_{Z H_{2}} G_{n}^{*}$ is stably permutation as a $Z N$-module. Now adding $W_{p}^{*}$ to both sides of

$$
Z_{p} N \otimes_{Z_{p} H C_{2}}\left(G_{n}^{*}\right)_{p}=\left(G_{n}^{*}\right)_{p} \oplus\left(G_{n}\right)_{p},
$$

we get by Lemma 2.8

$$
Z_{p} N \otimes_{Z_{p} H C_{2}}\left(G_{n}^{*}\right)_{p} \oplus W_{p}^{*} \cong\left(G_{n}\right)_{p} \oplus Z_{p} N^{k} \oplus Z_{p} N / H .
$$

Since $Z N^{k} \oplus Z N / H$ and $Z N \otimes_{Z H C_{2}}\left(G_{n}^{*}\right) \oplus W^{*}$ are $Z C$-free by Lemma 2.8, Theorem 2.7 implies that $\mathbf{C}\left(G_{n} \oplus Z G^{k} \oplus Z G / H\right)$ and $\mathbf{C}\left(Z G / N \otimes W^{*} \oplus Z G / H C_{2} \otimes G_{n}^{*}\right)$ are stably equivalent as $G$-fields. By Lemma $2.1, \mathbf{C}\left(G_{n}\right)$ is stably equivalent to $\mathbf{C}\left(Z G / N \otimes W^{*}\right)$ as $G$-fields. Now $\phi\left(A^{*}\right)=\left[G_{n}^{*}\right]=\Phi\left(W^{*}\right)$, hence $\mathbf{C}\left(Z G / N \otimes W^{*}\right)$ and $\mathbf{C}\left(Z G / N \otimes A^{*}\right)$ are stably equivalent as $G$-fields by Lemma 2.1. By Corollary 2.5 these fields are stably equivalent to $\mathbf{C}\left(A^{*}\right)$. Therefore by Theorem 2.6, $C_{n}$ is stably isomorphic to $\mathbf{C}\left(A^{*}\right)^{G}$ which is rational over $\mathbf{C}$, generated by the $(n-1)$-st elementary symmetric function [BL].

Theorem 3.4. The center of the ring of $7 \times 7$ generic matrices is stably rational over $\mathbf{C}$.

Proof. The proof is similar to that of $p=5$. Here $p-1=6$.

$$
\widehat{Z}_{p} N / N C_{3} \cong \widehat{Z}_{p} \oplus Z_{2} \oplus Z_{4}
$$

so

$$
\widehat{Z}_{p} N / H C_{3} \otimes \widehat{A}_{p}^{*} \oplus A_{2} \oplus A_{4} .
$$

Tensoring by $\widehat{A}_{p}^{*}$ we get by Theorem 3.2 and [CR1, Theorem 30.17]

$$
Z_{p} N / H C_{3} \otimes G_{n}^{*} \cong\left(G_{n}^{*} \oplus G_{n} \oplus B^{-}\right)_{p}
$$

since $A_{4} \cong \widehat{A}_{p}^{-}$. Now consider the exact sequence

$$
0 \rightarrow B^{-} \rightarrow Z N / H C_{2} \otimes B \rightarrow B \rightarrow 0 .
$$

This sequence splits at all primes different from 2 , and for the prime $2, \operatorname{Ext}_{N}^{1}\left(B_{2}, B_{2}^{-}\right)$ injects into $\operatorname{Ext}_{Z C_{3}}^{1}\left(B_{2}, B_{2}^{-}\right)=0$ since $B_{2}$ is $Z_{2} C$-free. So $Z N / H C_{2} \otimes B \cong B \oplus B^{-}$, 
and $B^{-}$is stably permutation. Also since $C_{3}$ is of order $2, Z N / H C_{3} \otimes G_{n}^{*}$ is stably permutation by [CT, Proposition 3, section 1]. Now adding $W_{p}^{*}$ to both sides of

$$
Z_{p} N \otimes_{Z_{p} H C_{3}}\left(G_{n}^{*}\right)_{p}=\left(G_{n}^{*}\right)_{p} \oplus\left(G_{n}\right)_{p} \oplus B_{p}^{-}
$$

we get by Lemma 2.8

$$
Z_{p} N \otimes_{Z_{p} H C_{3}}\left(G_{n}^{*}\right)_{p} \oplus W_{p}^{*} \cong\left(G_{n}\right)_{p} \oplus Z_{p} N^{k} \oplus Z_{p} N / H \oplus B_{p}^{-} .
$$

Since $Z N^{k} \oplus Z N / H \oplus B^{-}$and $Z N \otimes_{Z H C_{3}}\left(G_{n}^{*}\right) \oplus W^{*}$ are $Z C$-free, Theorem 2.7 implies that $\mathbf{C}\left(G_{n} \oplus Z G^{k} \oplus Z G / H \oplus Z G / H \otimes B^{-}\right)$and $\mathbf{C}\left(Z G / N \otimes W^{*} \oplus Z G / H C_{3} \otimes\right.$ $\left.G_{n}^{*}\right)$ are stably equivalent as $G$-fields. The argument is now the same as for $p=5$.

\section{REFERENCES}

[B] E. Beneish, Invertible Modules, J. Algebra 128 (1990), 101-125. MR 91b:20006

[B1] - The Grothendieck ring of invertible modules over nilpotent groups, J. Algebra 159 (1993), 400-418. MR 94i:20009

[BK] K. Brown, Cohomology of groups, Springer-Verlag, New York, 1982. MR 83k:20002

[BL] C. Bessenrodt and L. Le Bruyn, Stable rationality of certain PGL $L_{n}$-quotients, Invent. Math. 104 (1991), 179-199. MR 92m:14060

[CT] J.-L. Colliot-Thelene et J.-J. Sansuc, La R-equivalence sur les tores, Ann. Sci. Ecole Norm. Sup. (4) 10 (1977), 175-230. MR 56:8576

[CR1] C. W. Curtis and I. Reiner, Methods of Representation Theory, vol. 1, Wiley, New York, 1981. MR 90k:20001

[CR2] —_ Methods of Representation Theory, vol. 2, Wiley, New York, 1987. MR 88f:20002

$[\mathrm{EM}]$ S. Endo and T. Miyata, On the projective class group of finite groups, Osaka J. Math. 13 (1976), 109-122. MR 53:13315

[F1] E. Formanek, The center of the ring of $3 \times 3$ generic matrices, Linear and Multilinear Algebra 7 (1979), 203-212. MR 80h:16019

[F2] The center of the ring of $4 \times 4$ generic matrices, J. Algebra 62 (1980), 304-319. MR 81g:15032

[L] H. W. Lenstra, Rational functions invariant under a finite abelian group, Invent. Math. 25 (1974), 299-325. MR 50:289

[LL] L. Le Bruyn, Centers of generic division algebras, the rationality problem 1965-1990, Israel Journal of Math. 76 (1991), 97-111. MR 93f:16024

[P] C. Procesi, Non-commutative affine rings, Atti Accad. Naz. Lincei 8 (1967), 237-255. MR 37:256

[SD] D. Saltman, Retract rational fields and cyclic Galois extensions, Israel J. Math. 47 (1984), 165-215. MR 85j:13008

[SR] R. Swan, Invariant rational functions and a problem of Steenrod, Invent. Math. 7 (1969), 148-158. MR 39:5532

[S] J. Sylvester, On the involution of two matrices of second order, Southport: British Assoc. Report (1883), 430-432.

Department of Mathematics, University of Wisconsin-Parkside, Kenosha, Wisconsin $53141-2000$ 